\title{
Measurement of retinal nerve fibre layer by scanning laser polarimetry and high pass resolution perimetry in normal tension glaucoma with relatively high or low intraocular pressure
}

\author{
Motohiro Shirakashi, Shigeo Funaki, Haruko Funaki, Kiyoshi Yaoeda, Haruki Abe
}

\begin{abstract}
Aims-To determine whether any differences may exist in the relation between the neural capacity as determined by high pass resolution perimetry and the thickness of the retinal nerve fibre layer (RNFL) in patients having normal tension glaucoma (NTG) with a relatively high intraocular pressure (IOP) between 16 and $21 \mathrm{~mm}$ Hg (HNTG) $v$ those with a lower IOP below $15 \mathrm{~mm}$ Hg (LNTG).

Methods-Scanning laser polarimetry and high pass resolution perimetry were performed in 20 eyes of 20 patients with HNTG and 21 eyes of 21 patients with LNTG. The correlation between total and regional thickness of the peripapillary RNFL and the corresponding total and regional neural capacity with linear regression analysis were evaluated.
\end{abstract}

Results-Overall, although the total RNFL thickness was not significantly correlated with the total neural capacity, the RNFL thickness in each of the superior and inferior quadrants was significantly correlated with the corresponding regional neural capacity $(r=0.44, p=0.0045$; $r=0.39, p=0.0126$ for each). The RNFL thickness in each of the superior and inferior quadrants in the HNTG group was significantly correlated with the corresponding regional neural capacity $(r=0.52$, $p=0.0196 ; r=0.49, p=0.0286$ for each). No significant correlation between neural capacity and the RNFL thickness was observed either globally or regionally in the LNTG group.

Conclusion-The degree of the correlation between neural capacity as determined by high pass resolution perimetry and thickness of the RNFL as measured by scanning laser polarimetry appeared to differ in NTG patients with an IOP higher than $15 \mathrm{~mm} \mathrm{Hg} v$ those with a lower IOP. (Br F Ophthalmol 1999;83:353-357)

Scanning laser polarimetry has recently been used for the quantitative in vivo evaluation of the thickness of the retinal nerve fibre layer (RNFL). ${ }^{1-4}$ Such measurements are objective, rapid, and highly reproducible. ${ }^{34}$ High pass resolution perimetry is a method for evaluating the spatial resolution threshold that allows the calculation of a unique index termed the neural capacity (functional channel fraction or func- tional channels).$^{5-8}$ Neural capacity is thought to be an expression of the number of functioning retinal ganglion cells, and therefore provides an estimate of the number of viable nerve fibres in the retina. ${ }^{78}$

Few studies thus far have evaluated glaucoma patients for the correlation between neural capacity, as determined by high pass resolution perimetry, and the thickness of RNFL, as evaluated by scanning laser polarimetry. We previously studied the correlation between neural capacity and the thickness of RNFL in patients with primary open angle glaucoma (POAG) or normal tension glaucoma (NTG), and reported that the neural capacity was significantly correlated with the thickness of the RNFL in patients with POAG, but not in patients with NTG. ${ }^{9}$

Yamagami et $a l^{10}$ studied the influence of intraocular pressure (IOP) on the visual field in NTG, and reported that visual field damage was more dependent on the IOP in patients with a higher IOP (between 15 and $21 \mathrm{~mm} \mathrm{Hg}$ ) than in those with a lower IOP. In addition, Wang et $a l^{11}$ reported that patients with NTG who had a relatively low IOP below $15 \mathrm{~mm} \mathrm{Hg}$ exhibited a significantly greater area of peripapillary atrophy inferior to the optic disc than did patients with NTG having a relatively high IOP between 16 and $21 \mathrm{~mm} \mathrm{Hg}$. Such findings raise a question about the possibility of differing mechanisms of visual field or optic nerve damage in the two NTG groups classified according to the IOP. We now measured neural capacity by high pass resolution perimetry and thickness of the RNFL by scanning laser polarimetry in patients with NTG who had a relatively high or low IOP, and investigated whether any differences existed between the two groups in the correlation between neural capacity and thickness of the RNFL. Previous studies reported that the loss of visual field and damage to the optic nerve tended to be more localised in patients with NTG in contrast with those with POAG. ${ }^{12-14}$ The present study evaluated the correlation between neural capacity and thickness of the RNFL regionally as well as globally.

\section{Materials and methods}

This prospective study evaluated 41 eyes of 41 Japanese patients with NTG (Table 1). Each eye had a best corrected visual acuity of 0.8 or better, clear ocular media without any clinically significant cataract, open anterior chamber angle, a spherical equivalent refractive error
Accepted for publication 11 September 1998 
Table 1 Patient characteristics

\begin{tabular}{lllll}
\hline & & & & $\begin{array}{l}\text { HNTG } \\
\text { v LNTG } \\
\text { p Value }\end{array}$ \\
\hline Age (years) & Total $(n=41)$ & HNTG $(n=20)$ & LNTG $(n=21)$ & (13) \\
Sex ratio (male/female) & $18.4(13.8)$ & $52.5(15.1)$ & $54.3(12.7)$ & 0.6765 \\
Intraocular pressure (mm Hg) & $14.9(2.8)$ & $9 / 11$ & $9 / 12$ & 0.8901 \\
Refraction (dioptres) & $-0.49(2.71)$ & $-0.48(2.76)$ & $-0.50(2.73)$ & 0.9813 \\
Mean deviation (dB) & $-7.6(7.2)$ & $-6.5(7.2)$ & $-8.7(7.2)$ & 0.3499 \\
Disc area (mm $\left.{ }^{2}\right)$ & & & & \\
$\quad$ Total & $2.414(0.517)$ & $2.390(0.476)$ & $2.444(0.565)$ & 0.7414 \\
$\quad$ Superior quadrant & $0.614(0.129)$ & $0.610(0.130)$ & $0.617(0.132)$ & 0.8734 \\
$\quad$ Temporal quadrant & $0.590(0.136)$ & $0.580(0.117)$ & $0.599(0.154)$ & 0.6660 \\
$\quad$ Inferior quadrant & $0.616(0.132)$ & $0.610(0.130)$ & $0.623(0.137)$ & 0.7663 \\
$\quad$ Nasal quadrant & $0.598(0.138)$ & $0.589(0.116)$ & $0.606(0.159)$ & 0.6969 \\
\hline
\end{tabular}

HNTG = normal tension glaucoma with intraocular pressure between 16 and $21 \mathrm{~mm} \mathrm{Hg;} \mathrm{LNTG}$ $=$ normal tension glaucoma with intraocular pressure below $15 \mathrm{~mm} \mathrm{Hg}$.

$\star 30-2$ program of Humphrey visual field.

Data are expressed as mean (SD) except for sex ratio.

between -5 and +5 dioptres, and reliable measurements of visual field (fixation loss, false negative, and false positive of less than $20 \%$ ) performed on the $30-2$ program of the Humphrey visual field analyser (AllerganHumphrey, San Leandro, CA, USA). Each eye exhibited typical glaucomatous cupping of the optic disc and visual field defects with the Humphrey 30-2 program. The IOP was below $21 \mathrm{~mm} \mathrm{Hg}$ on repeated measurements without therapy. Patients had no history of retrobulbar optic neuritis, anterior ischaemic optic neuropathy, or intracranial lesion, and had not undergone intraocular surgery. Criteria for visual field abnormalities were three or more adjacent points with $5 \mathrm{~dB}$ or greater loss and/or one or more points with $10 \mathrm{~dB}$ or greater loss from the age corrected normal reference value.

Patients were divided into two groups according to the IOP. The first group consisted of 20 eyes of 20 patients with a relatively high IOP between 16 and $21 \mathrm{~mm} \mathrm{Hg}$ (HNTG). The second group consisted of 21 eyes of 21 patients with a relatively low IOP below $15 \mathrm{~mm}$ Hg (LNTG). The characteristics of each group appear in Table 1. The design of the present study was approved by our institutional review board. Informed consent for participation was obtained from each patient.

The thickness of the RNFL was measured with a scanning laser polarimeter (Nerve Fibre Analyser II, version 2.1.15 alpha, Laser Diagnostic Technologies, San Diego, CA, USA). Its principles have been described elsewhere. ${ }^{1-4}$ Although the nerve fibre analyser I does not provide an absolute calibration for its measurements, in its newer version, the nerve fibre analyser II, the calibration problem has been dealt with. In brief, the instrument consists of a confocal scanning ophthalmoscope with a polarisation modulator, a cornea polarisation compensator, and a polarisation detection unit. Measurement of the thickness of the RNFL is based on the assumption that the RNFL possesses birefringent properties that change the state of polarisation of an illuminating laser beam. This change (retardation) can be measured by determining the phase shift between extraordinary and ordinary beams. The extent of retardation is linearly correlated with the thickness of the RNFL. The light source consists of a near infrared diode laser (wavelength $780 \mathrm{~nm}$ ) in which the state of polarisation is modulated. The polarised light penetrates the birefringent RNFL and is partially reflected from the deeper layer of the retina. It is separated from the illuminating light beam by a non-polarising beam splitter. The state of polarisation of the light is analysed by the polarisation detection unit. The electrical signal from the detector is digitised and stored in the memory of a personal computer for later analysis. A complete scan produces a retardation map that consists of $256 \times 256$ (65 536) pixels in which the thickness of the RNFL for each pixel is measured.

In the present study, measurements of the thickness of the RNFL were made in a field of view that measured $15 \times 15$ degrees. The patient's pupils were not dilated. The optic disc margin was approximated by a circle placed around the inner margin of the peripapillary scleral ring. The thickness of the RNFL was measured within a ring of 10 pixels width and 1.75 disc diameters. The ring was concentric with reference to the disc margin. Mean thickness of the RNFL was determined within the entire ring and in its four 90 degree quadrants (superior, temporal, inferior, and nasal). Three images of each eye were obtained. The average thickness of the RNFL within the ring of the three images was calculated and used in the analyses. In each eye, a peripapillary atrophy or abnormal fundus pigmentation was not observed within the ring. All analyses were performed by the same person. We reported elsewhere the reproducibility of the measurements obtained with this instrument: the mean coefficient of variation $((\mathrm{SD}) /$ mean $\times 100)$ of the three measurements was a total RNFL thickness of $3.9 \%$, and a thickness of the RNFL in the superior, temporal, inferior, and nasal quadrants of $8.0 \%, 8.4 \%, 6.2 \%$, and $7.8 \%$, respectively. ${ }^{15}$

We performed high pass resolution perimetry (Ophthimus System, version 3.0, HighTech Vision, Malmö, Sweden) as described in detail elsewhere..$^{5-8}$ In brief, by using ring-shaped high pass spatial frequency filtered targets of different sizes, high pass resolution perimetry determines the resolution threshold in each of 50 locations within the central 30 degrees of the visual field, excluding the central most 5 degrees. The test targets consist of a light ring with dark inner and outer borders, with a space average luminance equal to the background $\left(20 \mathrm{~cd} / \mathrm{m}^{2}\right)$ and a within target contrast of 0.25 . The present study measured neural capacity in order to evaluate the number of functioning retinal ganglion cells and viable nerve fibres. ${ }^{78}$ Neural capacity is expressed as a percentage of the normal average. The present study analysed results that showed a good reliability. The interval between the performance of high pass resolution perimetry and the scanning laser polarimetry was less than 1 month for each eye.

In the present study, we further calculated quadrant neural capacity indices that corresponded to the superior, temporal, inferior, and nasal quadrants of the RNFL. Kono et al ${ }^{16}$ described in detail the calculation of local 

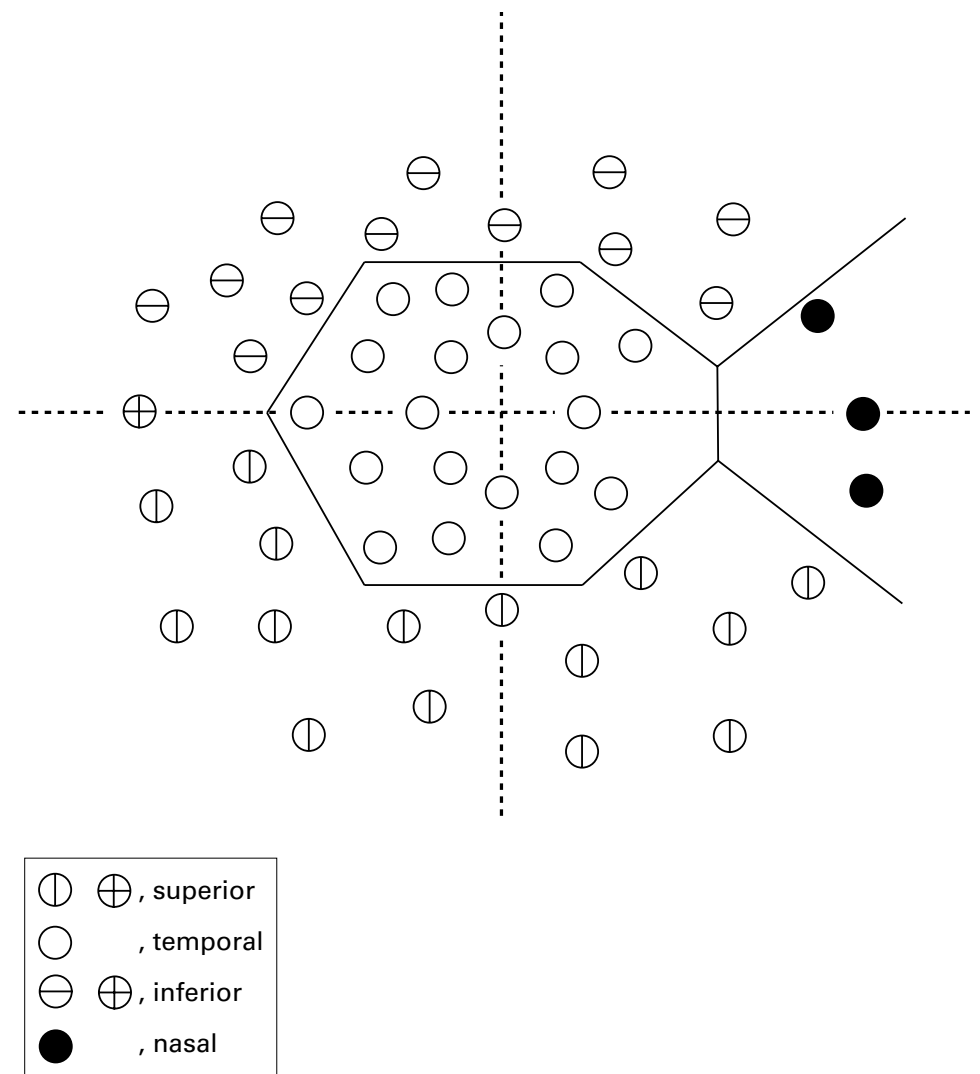

Figure 1 Divison of high pass resolution perimetry corresponding to the retinal nerve fibre layer in the superior, temporal, inferior, and nasal quadrants.

neural capacities at the 50 test locations and the four quadrant neural capacities of high pass resolution perimetry. As reported by those authors, ${ }^{16}$ we divided the visual field of high pass resolution perimetry into four sectors based on the proposal by Wirtschafter et $a l,{ }^{17}$ and calculated the local neural capacity at each of the 50 test locations. The quadrant neural capacity was calculated as the sum of the local neural capacities corresponding to each RNFL quadrant (Fig 1).

In the present study, the area of the optic disc in each eye was also calculated using a confocal scanning laser ophthalmoscope (Heidelberg Retina Tomograph, version 1.11, Heidelberg Engineering GmbH, Heidelberg,

Table 2 Neural capacity and thickness of retinal nerve fibre layer

\begin{tabular}{|c|c|c|c|c|}
\hline & $\begin{array}{l}\text { Total }(n=41) \\
\text { Mean }(S D)\end{array}$ & $\begin{array}{l}\text { HNTG }(n=20) \\
\text { Mean }(S D)\end{array}$ & $\begin{array}{l}\text { LNTG }(n=21) \\
\text { Mean }(S D)\end{array}$ & $\begin{array}{l}\text { HNTG } \\
v \text { LNTG } \\
\text { p Value }\end{array}$ \\
\hline \multicolumn{5}{|l|}{ Neural capacity (\%) } \\
\hline Total & $58.1(23.1)$ & $57.5(25.0)$ & $58.8(21.8)$ & 0.8576 \\
\hline Superior* & $17.3(6.4)$ & $17.0(6.9)$ & $17.5(6.0)$ & 0.8164 \\
\hline Temporal $\dagger$ & $26.0(11.4)$ & $25.2(12.2)$ & $26.7(10.9)$ & 0.6805 \\
\hline Inferior $\ddagger$ & $11.6(6.4)$ & $12.1(6.8)$ & $11.0(6.1)$ & 0.5926 \\
\hline Nasal§ & $4.1(1.3)$ & $3.9(1.2)$ & $4.3(1.4)$ & 0.3504 \\
\hline \multicolumn{5}{|c|}{ Thickness of retinal nerve fibre layer $(\mu \mathrm{m})$} \\
\hline Total & $62.6(15.9)$ & $63.4(16.5)$ & $61.9(15.7)$ & 0.7710 \\
\hline Superior quadrant & $72.9(22.1)$ & $72.1(22.3)$ & $73.7(22.4)$ & 0.8222 \\
\hline Temporal quadrant & $52.1(23.9)$ & $52.8(24.0)$ & $51.4(24.4)$ & 0.8598 \\
\hline Inferior quadrant & $72.2(20.8)$ & $73.5(20.8)$ & $71.0(21.2)$ & 0.7057 \\
\hline Nasal quadrant & $52.3(13.3)$ & $54.0(13.4)$ & $50.7(13.2)$ & 0.4387 \\
\hline
\end{tabular}

HNTG = normal tension glaucoma with intraocular pressure between 16 and $21 \mathrm{~mm} \mathrm{Hg}$; LNTG $=$ normal tension glaucoma with intraocular pressure below $15 \mathrm{~mm} \mathrm{Hg}$.

${ }^{\star}$ Quadrant neural capacity corresponding to retinal nerve fibre layer in the superior quadrant. tQuadrant neural capacity corresponding to retinal nerve fibre layer in the temporal quadrant. $\ddagger$ Quadrant neural capacity corresponding to retinal nerve fibre layer in the inferior quadrant.

\Quadrant neural capacity corresponding to retinal nerve fibre layer in the nasal quadrant.
Germany). The methods for optic disc analyses and their reproducibility have been described elsewhere. ${ }^{18-20}$ A mean image was calculated from three images with a field of view that measured $10 \times 10$ degrees. The margin of the optic disc was approximated by a contour placed around the inner margin of the peripapillary scleral ring. We calculated the disc areas for four 90 degree quadrants (superior, temporal, inferior, and nasal) as well as the total disc area.

Linear regression analysis was used to calculate correlation coefficients. Statistical comparisons between groups utilised unpaired or paired $t$ tests (two tailed) or the $\chi^{2}$ test.

A level of $\mathrm{p}<0.05$ was considered to be statistically significant.

\section{Results}

The mean IOP was significantly higher in the HNTG group than in the LNTG group (Table 1). The two groups did not differ significantly as to age, sex ratio, refraction, mean deviation of Humphrey visual field (30-2 program), and area of optic disc.

The two groups showed no significant difference in the total neural capacity (Table 2 ), and no significant differences in each of the four quadrant neural capacities. There was no significant difference in the total thickness of the RNFL, nor in the thickness of the RNFL in each of the four quadrants, between the two groups.

In all 41 eyes, there was no significant difference in thickness of the RNFL between the superior and inferior quadrants $(p=0.8258)$ (Table 2). In each group, there was also no significant difference in the thickness of the RNFL between the superior and inferior quadrants $(p=0.7605$, HNTG group; $\mathrm{p}=0.5611$, LNTG group). However, in all 41 eyes, the quadrant neural capacity that corresponded to the RNFL in the inferior quadrant was significantly lower than that which corresponded to the RNFL in the superior quadrant $(\mathrm{p}<0.0001)$. This was also true for each group $(p<0.0001$ for each). No significant correlation was observed between the total neural capacity and the total RNFL thickness in the total 41 eyes (Table 3). The thickness of the RNFL in each of the superior and inferior quadrants was significantly correlated with the corresponding quadrant neural capacity. However, the thickness of the RNFL in the temporal or nasal quadrant was not significantly correlated with the corresponding quadrant neural capacity.

The HNTG group exhibited no significant correlation between the total neural capacity and the total thickness of the RNFL (Table 3). The thickness of the RNFL in each of the superior and inferior quadrants showed a significant correlation with the corresponding quadrant neural capacity. However, no significant correlation was found between the thickness of the RNFL in the temporal or nasal quadrant and the corresponding quadrant neural capacity.

No significant correlation was observed between the total neural capacity and the total RNFL thickness in the LNTG group (Table 3). 
Table 3 Correlation between neural capacity and thickness of retinal nerve fibre layer

\begin{tabular}{|c|c|c|c|c|c|c|c|c|c|}
\hline & \multicolumn{3}{|c|}{ Total $(n=41)$} & \multicolumn{3}{|c|}{$H N T G(n=20)$} & \multicolumn{3}{|c|}{$\operatorname{LNTG}(n=21)$} \\
\hline & $r$ & Regression line & $p$ Value & $r$ & Regression line & p Value & $r$ & Regression line & $p$ Value \\
\hline Total $^{\star}$ & 0.29 & $\mathrm{Y}=51.06+0.20 \mathrm{X}$ & 0.0662 & 0.44 & $\mathrm{Y}=46.92+0.29 \mathrm{X}$ & 0.0555 & 0.13 & $\mathrm{Y}=56.53+0.09 \mathrm{X}$ & 0.5818 \\
\hline Superior† & 0.44 & $\mathrm{Y}=47.03+1.50 \mathrm{X}$ & 0.0045 & 0.52 & $\mathrm{Y}=43.64+1.67 \mathrm{X}$ & 0.0196 & 0.35 & $\mathrm{Y}=51.28+1.28 \mathrm{X}$ & 0.1239 \\
\hline Temporal $\ddagger$ & 0.02 & $\mathrm{Y}=50.83+0.05 \mathrm{X}$ & 0.8879 & 0.26 & $\mathrm{Y}=40.15+0.50 \mathrm{X}$ & 0.2787 & -0.22 & $\mathrm{Y}=64.30-0.48 \mathrm{X}$ & 0.3470 \\
\hline Inferior\$ & 0.39 & $\mathrm{Y}=57.71+1.26 \mathrm{X}$ & 0.0126 & 0.49 & $\mathrm{Y}=55.46+1.49 \mathrm{X}$ & 0.0286 & 0.27 & $\mathrm{Y}=60.46+0.96 \mathrm{X}$ & 0.2297 \\
\hline Nasal \| & 0.07 & $Y=49.33+0.72 X$ & 0.6492 & 0.14 & $\mathrm{Y}=48.03+1.52 \mathrm{X}$ & 0.5535 & 0.05 & $\mathrm{Y}=48.55+0.51 \mathrm{X}$ & 0.8149 \\
\hline
\end{tabular}

HNTG = normal tension glaucoma with intraocular pressure between 16 and $21 \mathrm{~mm} \mathrm{Hg}$; LNTG = normal tension glaucoma with intraocular pressure below $15 \mathrm{~mm}$ Hg.

*Total thickness of retinal nerve fibre layer $(\mathrm{Y}) v$ total neural capacity $(\mathrm{X})$.

†Thickness of retinal nerve fibre layer in the superior quadrant $(\mathrm{Y}) v$ the corresponding quadrant neural capacity $(\mathrm{X})$.

$\ddagger$ Thickness of retinal nerve fibre layer in the temporal quadrant $(\mathrm{Y}) v$ the corresponding quadrant neural capacity (X).

$\$$ Thickness of retinal nerve fibre layer in the inferior quadrant $(\mathrm{Y}) v$ the corresponding quadrant neural capacity $(\mathrm{X})$.

$\|$ Thickness of retinal nerve fibre layer in the nasal quadrant $(\mathrm{Y}) v$ the corresponding quadrant neural capacity (X).

Thickness of the RNFL in any of the four quadrants was not significantly correlated with the quadrant neural capacity.

\section{Discussion}

Although several investigators have reported a significant correlation between the indices of high pass resolution perimetry and optic disc topography in glaucomatous eyes, ${ }^{16}{ }^{21} 22$ others have found no such correlation. ${ }^{23}$ In patients with ocular hypertension or high tension glaucoma, Airaksinen et $a l^{21}$ reported that an index determined by high pass resolution perimetry, functional channel fraction (neural capacity), was significantly correlated with the severity of the RNFL abnormality, as determined by semiquantitative estimates, and with the area of the neuroretinal rim of the optic nerve head.

There are few reports on the relation between quantitative measurements of the RNFL thickness and the results of high pass resolution perimetry in NTG. In evaluating 37 patients with NTG, Kono et al ${ }^{16}$ observed a significant correlation between total neural capacity and total neuroretinal rim area, as measured by a confocal laser tomographic scanner. However, in our previous study of a relatively small number of patients with NTG $(n=23)$, we could not find a significant correlation between neural capacity and the thickness of RNFL, as measured by scanning laser polarimetry. ${ }^{9}$ In the present study, which included a larger number of patients with NTG $(n=41)$, although the total neural capacity tended to decrease with decreasing total RNFL thickness, the correlation did not reach statistical significance $(r=0.29, \mathrm{p}=0.0662)$.

In the patients with NTG studied by Kono et $a l,{ }^{16}$ the inferior neuroretinal rim area was significantly correlated with the corresponding quadrant neural capacity. However, those authors did not find any significant correlation between the superior neuroretinal rim area and the corresponding quadrant neural capacity. ${ }^{16}$ In the eyes evaluated in the present study, the thickness of the RNFL in the superior quadrant as well as in the inferior quadrant showed a significant correlation with the corresponding quadrant neural capacity. The correlations between the thickness of the RNFL in the superior and inferior quadrants and the corresponding quadrant neural capacities were stronger than the correlation between total thickness of the RNFL and total neural capac- ity. This is consistent with the recent report by Tarek et $a l^{24}$ who reported that the correlation between measurements of optic disc topography in the superior and inferior sectors and inferior and superior visual field were stronger than that between total optic disc measurements and global visual field in NTG. Our findings indicate that, in patients with NTG, the thickness of the RNFL in the superior or inferior quadrants, as measured by scanning laser polarimetry, reflects to some degree the number of functioning ganglion cells or viable nerve fibres in the correponding retina, as evaluated by high pass resolution perimetry.

Kono et al ${ }^{16}$ found no significant correlation between the temporal or nasal neuroretinal rim area and the corresponding quadrant neural capacity in patients with NTG. Similarly, the present study showed no significant correlation between the thickness of the RNFL in the temporal or nasal quadrant and the corresponding quadrant neural capacity. Our results indicate that the degree of correlation between the regional thickness of the RNFL and the corresponding quadrant neural capacity may not be identical between the superior or inferior quadrant and the temporal or nasal quadrant. The lack of correlation in the nasal quadrant may be due to a low coverage of the visual field corresponding to the RNFL in the nasal quadrant (three test points of high pass resolution perimetry). Since there is a relatively greater risk of measurement error in determining the RNFL thickness in the temporal than in the three other quadrants, the lack of correlation in the temporal quadrant may be attributable to measurement error. ${ }^{15}$

Although we found no significant difference in the RNFL thickness between the superior and inferior quadrants, the quadrant neural capacity that corresponded to the RNFL in the inferior quadrant was significantly lower than that which corresponded to the RNFL in the superior quadrant in all eyes, and in each group as well. Our findings are thus consistent with those of Kono et al ${ }^{16}$ who reported that quadrant neural capacity corresponding to the inferior neuroretinal rim area was significantly lower than that corresponding to the superior neuroretinal rim area, despite the lack of significant differences between the superior and inferior neuroretinal rim areas.

Although the total neural capacity in the HNTG group tended to decrease with a 
decrease in the total RNFL thickness, the correlation did not reach statistical significance $(\mathrm{r}=0.435, \mathrm{p}=0.0555)$. However, the thickness of the RNFL in each of the superior and inferior quadrants showed a significant correlation with the corresponding quadrant neural capacity, despite the lack of a significant correlation in either the temporal or nasal quadrant. However, in the LNTG group, neural capacity was not significantly correlated with the RNFL thickness, either globally or regionally. The two groups showed no significant differences in age, sex ratio, or refraction. The total and individual quadrant neural capacity also did not differ significantly between the two groups. Although Weinreb et $a l^{3}$ recognised the influence of the blood vessels on RNFL measurements using scanning laser polarimetry, we did not exclude any polarimetric data related to the blood vessels in the present study. The blood vessels may make up a larger proportion of the peripapillary retina area in eyes with smaller discs. If retardation is reduced over blood vessels, ${ }^{3}$ the thickness of the RNFL measured within an optic disc size dependent ring may be lower in eyes with smaller discs compared with larger discs. However, the total and regional disc areas were similar in the HNTG and LNTG groups.

Our results suggest that the degree of correlation between the morphological changes in the regional RNFL, as measured by scanning laser polarimetry, and the functional changes in ganglion cells or nerve fibres in the corresponding retina, as evaluated by high pass resolution perimetry, were not identical in the HNTG and LNTG groups with progressive glaucomatous damage. The present study may provide indirect evidence to support the concept of a difference in the mechanism of glaucomatous damage to the RNFL in the HNTG and LNTG groups, each with a differing pathophysiology as well as visual prognosis. Although we evaluated the relation between functional and morphological changes in the RNFL regionally as well as globally, the method used in the present study may not have been sufficient to detect the presence of more localised abnormalities in the RNFL. It is possible that the presence of such localised morphological and/or functional changes in the RNFL of the LNTG group, compared with the HNTG group, might not have been detected, even by the method of regional analysis used in the present study.

Weinreb et al ${ }^{1}$ reported an excellent correlation between retardation as measured by scanning laser polarimetry and the histopathological measurement of RNFL thickness in monkey eyes. Scanning laser polarimetry provides quantitative measurements that correspond to known properties of the RNFL in normal and glaucomatous human eyes. ${ }^{3}$ However, a recent histological study of normal human eyes reported several differences between the histological thickness of the RNFL and the results obtained by scanning laser polarimetry. ${ }^{25}$ Thus, whether scanning laser polarimetry effectively and accurately measures the thickenss of the RNFL in human eyes requires further study. Our findings need to be confirmed in a study of a larger number of patients. The authors have no financial, commercial, or proprietary
interest in any of the products or companies mentioned in this interest
study.

The authors wish to thank Lars Frisén, MD, PhD The authors wish to thank Lars Frisén, MD, PhD
(Department of Ophthalmology, University of Gothenburg, Sweden) for providing the software for calculating the local neural capacity.

1 Weinreb RN, Dreher AW, Coleman A, et al. Histopathologic validation of Fourier-ellipsometry measurements of retinal nerve fiber layer thickness. Arch Ophthalmol 1990;108:55760.

2 Dreher AW, Reiter K, Weinreib RN. Spatially resolved birefringence of the retinal nerve fiber layer assessed with a retinal laser ellipsometer. Appl Optics 1992;31:3730-5.

3 Weinreb RN, Sakiba S, Zangwill L. Scanning laser polarimetry to measure the nerve fiber layer of normal and glaucomatous eyes. Am f Ophthalmol 1995;119:627-36.

matous eyes. Am f Ophthalmol 1995;119:627-36.
4 Poinoosawmy D, Fontana L, Wu JX, et al. Variation of nerve Poinoosawmy D, Fontana L, Wu JX, et al. Variation of nerve
fibre layer thickness measurement with age and ethnicity by fibre layer thickness measurement with age and ethnicity by 4.

5 Frisén L. A computer-graphics visual field screener using high-pass spatial frequency resolution targets and multiple feedback devices. Doc Ophthalmol Proc Ser 1987;49:441-6.

6 Frisén L. High-pass resolution targets in peripheral vision. Ophthalmology 1987;94:1104-8.

7 Frisén L. Acuity perimetry: estimation of neural channels. Int Ophthalmol 1988;12:169-74.

8 Frisén L. High-pass resolution perimetry: recent developments. In: Heil A, ed. Perimetry update 1988/1989. Amsterdam: Kugler and Ghedini, 1989:369-75.

9 Shirakashi M, Abe H, Sawaguchi S, et al. Measurement of thickness of retinal nerve fiber layer by scanning laser polarimetry and high-pass resolution perimetry in patients with primary open-angle or normal-tension glaucoma. Acta Ophthalmol Scand 1997;75:641-4

10 Yamagami J, Shirato S, Araie M. The influence of the intraocular pressure on the visual field of low tension glaucoma. Acta Soc Ophthalmol fpn 1990;94:514-8.

11 Wang XH, Stewart WC, Jackson GJ. Difference in optic discs in low-tension glaucoma patients with relatively low or high pressures. Acta Ophthalmol Scand 1996;74:364-7.

12 Levene RZ. Low-tension glaucoma: a critical review and new material. Surv Ophthalmol 1980;24:621-64.

3 Caprioli J, Spaeth GL. Comparison of visual field defects in the low-tension glaucomas with those in the high-tension glaucomas. Am fo Ophthalmol 1984;97:730-7.

14 Drance SM, Douglas GR, Airaksinen PJ, et al. Diffuse visual field loss in chronic open-angle and low-tension glaucoma. Am f Ophthalmol 1987; 104:577-80.

15 Shirakashi M, Abe H, Sawaguchi S. Relationship between retinal nerve fiber layer thickness and visual field loss in glaucoma. Fournal of the Eye (Atarashii Ganka) 1996;13: 597-9.

16 Kono Y, Chi QM, Tomita G, et al. High-pass resolution permetry and a Humphrey field analyzer as indicators of glaucomatous optic disc abnormalities: a comparative study. Ophthalmology 1997;104:1496-502.

17 Wirtschafter JD, Becker WL, Howe JB, et al. Glaucoma visual field analysis by computed profile of nerve fiber function in optic disc sectors. Ophthalmology 1982;89:25567.

18 Mikelberg FS, Wijsman K, Schultzer M. Reproducibility of topographic parameters obtained with the Heidelberg retina tomograph. F Glaucoma 1993;2:101-3.

19 Lusky M, Bosem ME, Weinreb RN. Reproducibility of optic nerve head topography measurements in eyes with undilated pupils. F Glaucoma 1993;2:104-9.

20 Rohrschneider K, Burk ROW, Kruse FE, et al. Reproducibility of the optic nerve head topography with a new laser tomographic scanning device. Ophthalmology 1994;101: tomographi.

21 Airaksinen PJ, Tuulonen A, Välimäki, et al. Retinal nerve fiber layer abnormalities and high-pass resolution perimfiber layer abnormalities and high-pass resolution

22 Lachenmayr BJ, Airaksinen PJ, Drance SM, et al. Correlation of retinal nerve-fiber-layer loss, changes at the optic nerve head and various psychophysical criteria in glaucoma. Graefes Arch Clin Exp Ophthalmol 1991;229:133-8.

23 Chauhan BC, LeBlanc RP, McCormick TA, et al. Correlation between the optic disc and results obtained with conventional, high-pass resolution and pattern discrimination perimetry in glaucoma. Can $\mathcal{F}$ Ophthalmol 1993;28:312-6.

24 Tarek EE, Spaeth GL, Moster RM, et al. Quantitative difference between the optic nerve head and peripapillary retina in low- tension and high-tension primary open-angle glaucoma. Am 7 Ophthalmol 1997;124:805-13.

25 Varma R, Skaf M, Barron E. Retinal nerve fiber layer thickness in normal human eyes. Ophthalmology 1996;103: $2114-9$. 